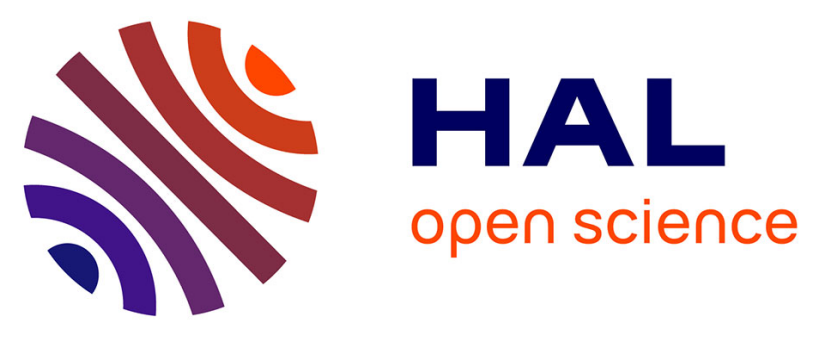

\title{
Lyapunov Stability Analysis of Switching Controllers in Presence of Sliding Modes and Parametric Uncertainties With Application to Pneumatic Systems
}

\author{
Omar Ameur, Paolo Massioni, Gérard Scorletti, Xavier Brun, Mohamed \\ Smaoui
}

\section{To cite this version:}

Omar Ameur, Paolo Massioni, Gérard Scorletti, Xavier Brun, Mohamed Smaoui. Lyapunov Stability Analysis of Switching Controllers in Presence of Sliding Modes and Parametric Uncertainties With Application to Pneumatic Systems. IEEE Transactions on Control Systems Technology, 2016, 26 (6), pp.1953-1964. 10.1109/TCST.2016.2529964 . hal-01281630

\author{
HAL Id: hal-01281630 \\ https://hal.science/hal-01281630
}

Submitted on 20 Mar 2019

HAL is a multi-disciplinary open access archive for the deposit and dissemination of scientific research documents, whether they are published or not. The documents may come from teaching and research institutions in France or abroad, or from public or private research centers.
L'archive ouverte pluridisciplinaire HAL, est destinée au dépôt et à la diffusion de documents scientifiques de niveau recherche, publiés ou non, émanant des établissements d'enseignement et de recherche français ou étrangers, des laboratoires publics ou privés. 


\title{
Lyapunov stability analysis of switching controllers in presence of sliding modes and parametric uncertainties with application to pneumatic systems
}

\author{
Omar Ameur, Paolo Massioni, Gérard Scorletti, Xavier Brun and Mohamed Smaoui
}

\begin{abstract}
This paper concerns the control and the stability analysis of pneumatic actuators, which are nowadays of widespread use in the industry. A problem related to the use of such actuators is the so-called "stick-slip", due to the presence of dry friction in the system. In this paper we provide an empirical switching control law which avoids this phenomenon, as well as a general approach to the stability analysis of nonlinear systems which will let us prove the stability of the closed-loop system. The approach is based on casting the closed-loop system into a piecewise-affine form and finding a Lyapunov function for it. Such an approach will be able to cope with the special features of the controlled pneumatic system model, namely the presence of sliding modes, a whole equilibrium set and uncertainties on the values of a few parameters.

At the end of the paper we will show how such a method can be successfully applied to our experimental setup under several different hypotheses.
\end{abstract}

Index Terms-Piecewise-affine systems, piecewise-polynomial Lyapunov functions, switched control, fluid power.

\section{INTRODUCTION}

Electropneumatic systems are widely used in industry, especially in the form of pneumatic actuators or cylinders. Their efficient use depends on good control laws, which are often non trivial to synthesize due to nonlinear dynamics. In particular, friction is a phenomenon which plays a key role in the dynamic behavior of pneumatic cylinders, as the presence of friction may lead to what is called "stick-slip" [20], [7]. Such a phenomenon typically occurs in the presence of dry friction combined with an integration effect (either in the control law or in the system itself), and it consists, in pneumatic cylinders, in a displacement of the rod a while after it has come to a rest; this is due to the fact that the force acting on the rod initially becomes smaller that the threshold which is necessary for a motion in presence of dry friction, but later on this threshold is overcome due to a slowly growing integrator. For example, Figure 1 features the record of an experiment on a pneumatic cylinder showing an occurrence of stick-slip. We can see that the rod, following the given reference, comes to a rest in the first half second; then it starts moving again, with no changes for the setpoint.

In this case, stick-slip is caused by the presence of dry friction and by the pressure dynamics in the chambers, which continue to evolve (integrating the net incoming mass flow from the servovalves) even after the rod has stopped.

O. Ameur and G. Scorletti are with Laboratoire Ampère, UMR CNRS 5005, École Centrale de Lyon, Université de Lyon, \{omar.ameur, gerard.scorletti\}@ec-lyon. fr

P. Massioni, X. Brun and M. Smaoui are with Laboratoire Ampère, UMR CNRS 5005, INSA de Lyon, Université de Lyon, \{paolo.massioni, xavier.brun, mohamed.smaoui\}@insa-lyon.fr

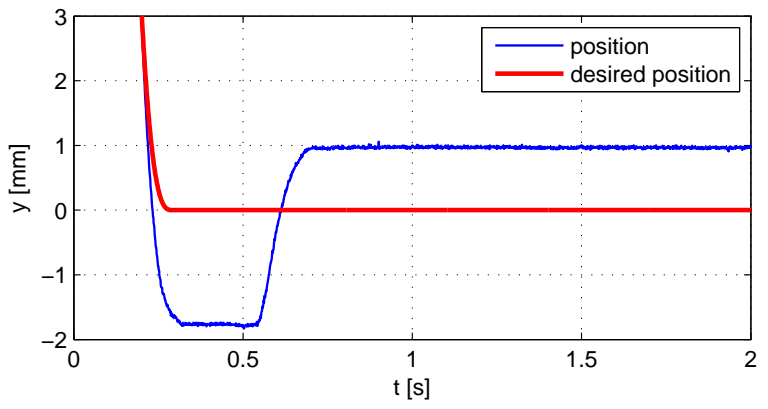

Fig. 1. Stick-slip phenomenon on a pneumatic cylinder.

To avoid this highly undesirable phenomenon, a control law has been proposed recently in [28]. This solution consists in a classic feedback linearization, which compensates for the nonlinearities in the pneumatic model, together with an appropriate switching law. The controller switches from a trajectory tracking control to a pressure control after the rod has come to a rest, offsetting the pressure difference between the two chambers and avoiding an uncontrolled evolution that eventually could make the rod restart. A major problem with this solution, which has been otherwise verified as very effective in the practice, was to find a formal proof of its stability; in fact, such a proof has not been given in the original reference [28].

The difficulty in finding a formal proof of stability lies in the pressure dynamics of the pneumatic chambers, and in the presence of dry friction, which make the electropneumatic actuator a strongly non-linear system. Typically, feedback linearization can be used to cancel the nonlinearities with a feedback control, with the "caveat" that an uncertainty in the model can lead to catastrophic effects. In this study we will analyze a control law, based on the one in [28], which cancels all the nonlinear effects through feedback linearization with the exception of the most difficult to model quantitatively, namely the friction. We will then approach the problem of proving the stability of the closed-loop system by casting it into a piecewise-affine (PWA) form [12], [23], [25], [22]. In order to do that, we will consider two different models for the dry friction; we will consider both a simplified model, where the friction force is a continuous function of the velocity of the rod (in a so-called "saturation" shape), as well as a more realistic, discontinuous model of friction. In fact, as indicated by many works [3], [11], [29], [9], more accurate models of friction feature discontinuities, which can lead to the appearance of sliding modes [14], [13] in the system dynamics. 
In the literature, we can find several approaches for dealing with sliding modes in piecewise-affine systems [18], [12], [5], [16], [26] but unfortunately, none of these approaches are enough to deal with the stability of our pneumatic actuator systems. First of all, we have verified that searching for a piecewise-quadratic Lyapunov functions is too conservative and does not yield any valid solution. Secondly, these former methods are not able to cope with the presence of a whole equilibrium set (not only a point) in a sliding mode. In this work, we propose new sufficient conditions for stability based on piecewise-polynomial Lyapunov functions, which can be given in terms of linear matrix inequalities (LMIs) and linear matrix equalities (LMEs). These conditions are also able to cope with the uncertainties in the friction models, and we have used them successfully for proving the stability of the closed-loop system for all the possible models of friction.

Some of the results of this paper have been partially presented in [1], [2], which contain only two special cases of the main, more general result introduced here. This paper analyses all the possible cases and offers a unified point of view.

The remainder of this paper is organized as follows. Section II contains the description of the pneumatic actuator model with the proposed switching control law avoiding "stick-slip". Section III introduces the uncertain PWA class of systems and the tools that can be used for the robust stability analysis in the presence of sliding modes and parameters variations. Section IV contains the main theoretical result, i.e. a method for proving robust stability, whereas Section V shows its different applications to our test bench model. The conclusions are given in Section VI.

\section{Notation}

We denote by $\mathbb{R}$ the field of real numbers and by $\mathbb{R}^{n \times m}$ the set of real $n \times m$ matrices. Let $A^{T}$ indicate the transpose of a matrix $A$, and let $I$ be the identity matrix. We use also the notation $A \succ 0(A \prec 0)$ to indicate that all the eigenvalues of the symmetric matrix $A$ are strictly positive (negative). The symbol $\left(\begin{array}{l}n \\ k\end{array}\right)$ indicates the binomial coefficient, for which we have

$$
\left(\begin{array}{l}
n \\
k
\end{array}\right)=\frac{n !}{k !(n-k) !}
$$

\section{Pneumatic actuator Systems}

We consider a pneumatic cylinder test bench like the one shown in Figure 2. The setup is intended for applications in rectilinear motion [8], [6], [27]. It comprises an actuator in the form of a pneumatic cylinder (double acting) with a rod connected to a carriage on rails. The actuator is powered by compressed air, with two servovalves for controlling the flow supplied to both the chambers of the cylinder. Two sensors measure the pressures in the chambers.

Based on [28], the physical model of the system in open loop is given by the following equations (see Table I for the notation):

\begin{tabular}{|ll|}
\hline$y$ & position of the piston rod $(\mathrm{m})$ \\
$v$ & velocity (m $\left.\mathrm{s}^{-1}\right)$ \\
$p_{P}, p_{N}$ & pressures in the cylinder chambers $(\mathrm{Pa})$ \\
$a$ & acceleration $\left(\mathrm{m} \mathrm{s}^{-2}\right)$ \\
$F_{s}$ & dry friction force $(\mathrm{N})$ \\
$k$ & gas polytropic constant \\
$l$ & length of stroke $(\mathrm{m})$ \\
$M$ & load (carriage and rod mass) $(\mathrm{kg})$ \\
$q_{m P}, q_{m} N$ & mass flow rate provided by the servovalves \\
$r$ & to the cylinder chambers $\left(\mathrm{kg} \mathrm{s}^{-1}\right)$ \\
$S$ & perfect gas constant $\left(\mathrm{J} \mathrm{kg}^{-1} \mathrm{~K}^{-1}\right)$ \\
$T$ & area of cylinder bore $\left(\mathrm{m}^{2}\right)$ \\
$V$ & temperature $(\mathrm{K})$ \\
$V_{D}$ & volume $\left(\mathrm{m}^{3}\right)$ \\
& dead volume of cylinder chamber $\left(\mathrm{m}^{3}\right)$ \\
\hline
\end{tabular}

TABLE I

NOTATION.

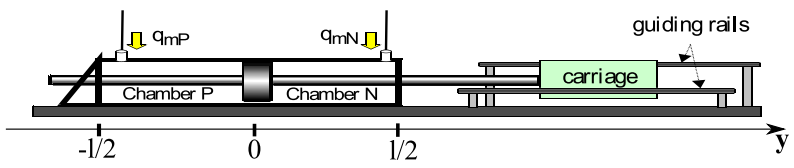

Fig. 2. Electro-pneumatic actuator.

$$
\left\{\begin{array}{l}
\dot{y}=v \\
\dot{v}=\frac{1}{M}\left(S\left(p_{P}-p_{N}\right)-F_{f}(v)\right) \\
\dot{p}_{N}=\frac{k r T}{V_{N}(y)}\left(\frac{S}{r T} p_{N} v+q_{m N}\right) \\
\dot{p}_{P}=\frac{k r T}{V_{P}(y)}\left(\frac{-S}{r T} p_{P} v+q_{m P}\right)
\end{array}\right.
$$

where the inputs are the two mass flow rates $q_{m P}$ and $q_{m N}$, and $V_{P}(y)=V_{0}+S y, V_{N}(y)=V_{0}-S y$ with $V_{0}=V_{D}+S \frac{l}{2}$. $F_{f}(v)$ is the dry friction force, whose nonlinear model is given in the literature by several relations [3], [11], [29], [9]. In our case, we consider two possible models of the dry friction forces, one in a continuous saturation form $\left(M_{2}\right)$ and another in a discontinuous, "relay" form $\left(M_{1}\right)$, both shown in Figure 3, such as:

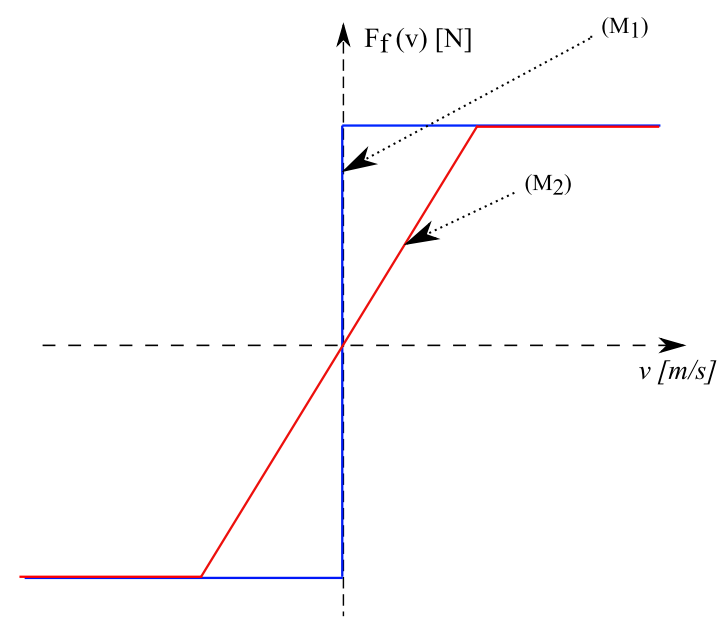

Fig. 3. Dry friction models. 


$$
M_{1}: \quad F_{f}(v) \begin{cases}=+F_{s} & \text { for } v>0 \\ \in\left[-F_{s},+F_{s}\right] & \text { for } v=0 \\ =-F_{s} & \text { for } v<0 .\end{cases}
$$

and

$$
M_{2}: \quad F_{f}(v)= \begin{cases}F_{s} & \text { for } v>\varepsilon \\ \frac{F_{s}}{\varepsilon} v & \text { for }-\varepsilon \leq v \leq \varepsilon \\ -F_{s} & \text { for } v<-\varepsilon\end{cases}
$$

In order to overcome the stick-slip phenomenon, we propose the following control law adapted from [28]. This law is based on a feedback linearization of the model in (1), and it switches between the two following modes.

\section{- Trajectory tracking law (\#1)}

$$
\left\{\begin{aligned}
q_{m P} & =\frac{V_{P}(y)}{k r T}\left[\frac{k S}{V_{P}(y)} v p_{P}+\dot{p}_{P d}-k_{P} e_{P}\right] \\
q_{m N} & =\frac{M V_{N}(y)}{S k r T}\left[\frac{S k r T}{M V_{P}(y)} q_{m P}+\frac{S^{2} k v}{M}\left(-\frac{p_{P}}{V_{P}(y)}-\frac{p_{N}}{V_{N}(y)}\right)\right. \\
& \left.\quad j_{d}+k_{a} e_{a}+k_{v} e_{v}+k_{y} e_{y}\right]
\end{aligned}\right.
$$

which allows the tracking of a given time-varying position reference; under the hypothesis of no state or input saturation, it yields the following closed-loop dynamics:

$$
\left\{\begin{array}{l}
\dot{e}_{y}=e_{v} \\
\dot{e}_{v}=e_{a f}-\frac{1}{M} F_{f}(v) \\
\dot{e}_{a f}=-k_{a} e_{a f}-k_{v} e_{v}-k_{y} e_{y}+\frac{k_{a}}{M} F_{f}(v) \\
\dot{e}_{P}=-k_{P} e_{P}
\end{array}\right.
$$

\section{- Pressure control law (\#2)}

$$
\left\{\begin{array}{l}
q_{m P}=\frac{V_{P}(y)}{k r T}\left[\frac{k S}{V_{P}(y)} v p_{P}+\dot{p}_{P d}-k_{P} e_{P}\right] \\
q_{m N}=\frac{V_{N}(y)}{k r T}\left[-\frac{k S}{V_{N}(y)} v p_{N}+\dot{p}_{N d}-k_{N} e_{N}\right]
\end{array}\right.
$$

which regulates the pressures in the two chambers in order to avoid stick-slip; this law is active when the carriage has arrived at the desired position, resulting in the following closed-loop dynamics:

$$
\left\{\begin{array}{l}
\dot{e}_{y}=e_{v} \\
\dot{e}_{v}=e_{a f}-\frac{1}{M} F_{f}(v) \\
\dot{e}_{a f}=\frac{S}{M}\left(k_{N}-k_{P}\right) e_{P}-k_{N} e_{a f} \\
\dot{e}_{P}=-k_{P} e_{P}
\end{array}\right.
$$

In all of the equations above, $e_{a f}=e_{a}+\frac{1}{M} F_{f}(v)$. $e_{y}, e_{v}, e_{a}, e_{P}, e_{N}$ are the errors between states and their desired values; the constants $k_{y}, k_{v}, k_{a}, k_{P}$ and $k_{N}$ are the state feedback gains chosen by a pole-placement on the feedback-linearized model. The switching criterion is

$$
\begin{aligned}
& \# 1 \rightarrow \# 2:\left|e_{y}\right| \leq \varepsilon_{1} \wedge\left|e_{v}\right| \leq \varepsilon_{2} \wedge\left|v_{d}\right| \leq \varepsilon_{3} \\
& \# 2 \rightarrow \# 1:\left|e_{y}\right|>\varepsilon_{1} \vee\left|e_{v}\right|>\varepsilon_{2} \vee\left|v_{d}\right|>\varepsilon_{3}
\end{aligned}
$$

where $\varepsilon_{1}, \varepsilon_{2}$ and $\varepsilon_{3}$ are small arbitrary constants. The above switching controller has shown no instability either in simulation or on the test bench and has never caused the occurrence of stick-slip. Figure 4 and Figure 5 show trajectories obtained using this control law, respectively in simulation and on the test bench; the system can switch to pressure control (\#2) only when the trajectory becomes constant.

Remark 1: The chosen control law is based on a feedback linearization that cancels out all the nonlinearities of the system but the ones caused by friction. We avoid canceling them because there is a high degree of uncertainty on the friction forces, so exact cancellation is impossible.

The topic of the rest of this paper is the formal proof of stability. One of the issues that we will need to face is that the stability of the system has to be proven for a special kind of convergence of the state, not to the setpoint, but to a whole set in its neighborhood. This is due to the well-known fact that moving systems subject to dry friction cannot be easily brought to a stop at a desired point, but they will rather stop in its proximity (this can be seen for example in Figure 4, when the rod stops just after $t=2 \mathrm{~s}$, as well as in Figure 5, where the error never goes exactly to 0 ).

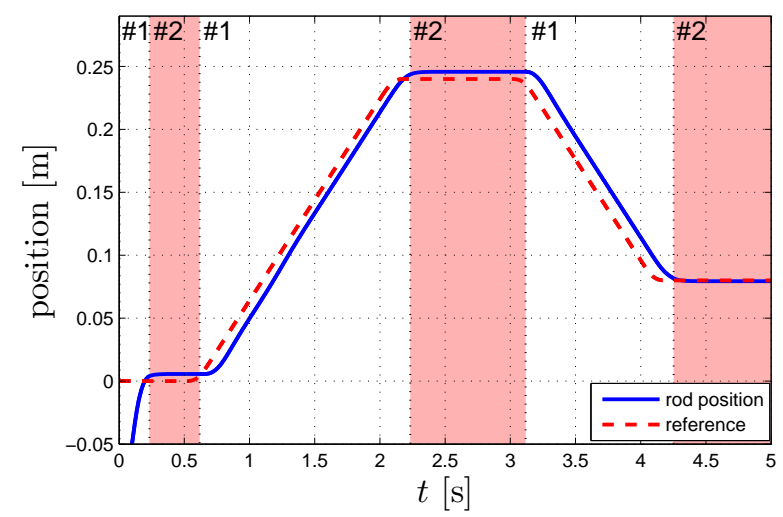

Fig. 4. Simulation of position evolution with respect to the desired position. The background colors are related to the active control mode (\#1 or \#2).
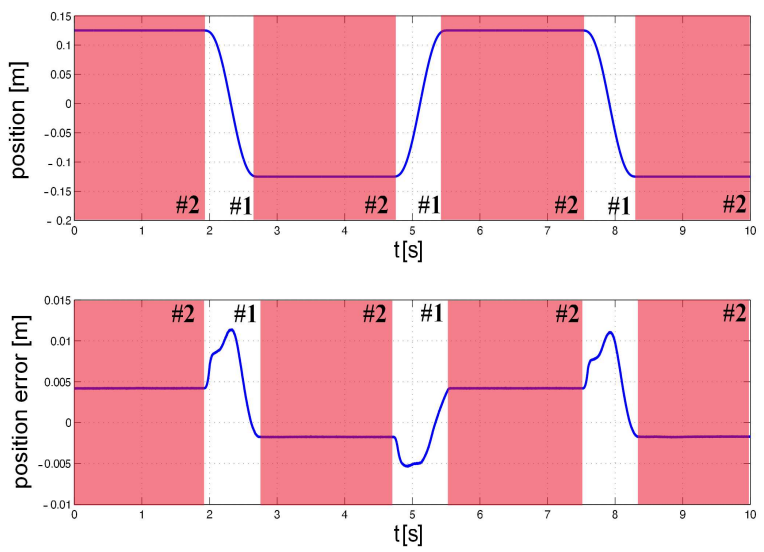

Fig. 5. Experimental results: evolution of the rod position and the postion error with respect to the desired reference. The background colors are related to the active control mode (\#1 or \#2). No stick-slip occurs.

\section{PROPOSED APPROACH}

Let us introduce a partition of $\mathbb{R}^{n}$ into $N$ polyhedral cells $X_{i}$ with disjoint interior, with $i \in \mathcal{I}$, a set of $N$ valid indices. 
We partition $\mathcal{I}=\mathcal{I}_{0} \cup \mathcal{I}_{1}$ (with $\mathcal{I}_{0} \cap \mathcal{I}_{1}=\emptyset$ ) such as $0 \in X_{i}$ if $i \in \mathcal{I}_{0}$, otherwise $0 \notin X_{i}$ if $i \in \mathcal{I}_{1}$ (the cells with index in $\mathcal{I}_{0}$ contain the origin).

Definition 2 (Piece-wise affine with polytopic uncertainties): A dynamical system is called "piecewise affine" (PWA) with polytopic uncertainties if it has the following dynamics [1], [19], [15]:

$$
\dot{x}(t)=\sum_{k=1}^{q} \lambda_{k}(t)\left(A_{i, k} x(t)+a_{i, k}\right), \text { for } x(t) \in X_{i}, i \in \mathcal{I}
$$

where $x(t) \in \mathbb{R}^{n}$ denotes the state-space vector and $\lambda_{k}(t)$ are continuously time-varying parameters which can take values according to

$$
\left\{\begin{array}{l}
\lambda_{k}(t) \geq 0 \forall k \in\{1, \ldots, q\} \\
\sum_{k=1}^{q} \lambda_{k}(t)=1
\end{array}\right.
$$

Equivalently, for $\bar{x}(t)^{T}=\left[x(t)^{T} 1\right]$

$$
\dot{\bar{x}}(t)=\sum_{k=1}^{q} \lambda_{k}(t) \underbrace{\left[\begin{array}{cc}
A_{i, k} & a_{i, k} \\
0 & 0
\end{array}\right]}_{\bar{A}_{i, k}} \bar{x}(t), \text { for } x(t) \in X_{i}, i \in \mathcal{I}
$$

The domain of each cell $X_{i}$ is described by matrices $\bar{E}_{i} \in$ $\mathbb{R}^{l_{i} \times(n+1)}$ such as:

$$
x \in X_{i} \Rightarrow \bar{E}_{i} \bar{x} \geq 0,
$$

and the boundaries between two cells $X_{i}$ and $X_{j}$ by matrices $\bar{F}_{i j} \in \mathbb{R}^{r_{i j} \times(n+1)}$ such as

$$
\left\{\begin{array}{l}
\Gamma=\left\{(i, j) \mid X_{i} \cap X_{j} \neq \emptyset\right\} \\
\forall(i, j) \in \Gamma, X_{i} \cap X_{j} \subseteq\left\{x \mid \bar{F}_{i j} \bar{x}=0\right\} .
\end{array}\right.
$$

We define $\lambda^{T}=\left[\begin{array}{lll}\lambda_{1} & \ldots & \lambda_{q}\end{array}\right]^{T}$. We then formulate a set of assumptions which will hold throughout the paper.

Assumption 1: A sliding mode may occur on any boundary in $\Gamma$ between two neighboring cells $X_{i}$ and $X_{j}$ (see Figure 6), i.e. for $x \in X_{i} \cap X_{j},(i, j) \in \Gamma$, we have the following dynamics (according to Filippov [13]):

$$
\begin{gathered}
\dot{\bar{x}}(t)=\left(\theta(\lambda) \sum_{k=1}^{q} \lambda_{k}(t) \bar{A}_{i, k}+(1-\theta(\lambda)) \sum_{k=1}^{q} \lambda_{k}(t) \bar{A}_{j, k}\right) \bar{x}(t), \\
0 \leq \theta(\lambda) \leq 1 .
\end{gathered}
$$

which means that the matrix describing the system dynamics at a boundary is a convex combination of the matrices of the neighboring cells.

Assumption 2: The set $E_{e q} \supseteq\{0\}$ of the equibrium points for (8) is a subset of $\bigcup_{i \in \mathcal{I}_{0}} X_{i}$ (notice that the equilibrium set might also be on a sliding mode).

Assumption 3: Two cases can be distinguished:

- if the origin is in the interior of a cell $X_{i}$ (for which we have then that $\left.\mathcal{I}_{0}=\{i\}\right): \sum_{k=1}^{q} \lambda_{k}(t) \bar{A}_{i, k}$ does not have any constant terms $\left(a_{i, k}=0\right.$, for $\left.k=1, \ldots, q\right)$; we define $\overline{\mathcal{A}}_{0}(\lambda)=\sum_{k=1}^{q} \lambda_{k}(t) \bar{A}_{i, k}$;

- if the origin is in the boundary between cells $X_{i}$ and $X_{y}$ : there exists a $\bar{\theta}(\lambda), 0 \leq \bar{\theta}(\lambda) \leq 1$, for which $\bar{\theta}(\lambda) \sum_{k=1}^{q} \underline{\lambda_{k}}(t) a_{i, k}+(1-\bar{\theta}(\lambda)) \sum_{k=1}^{q} \lambda_{k}(t) a_{j, k}=0 ;$ we define $\overline{\mathcal{A}}_{0}(\lambda)=\bar{\theta}(\lambda) \sum_{k=1}^{q} \lambda_{k}(t) \bar{A}_{i, k}+(1-$ $\bar{\theta}(\lambda)) \sum_{k=1}^{q} \lambda_{k}(t) \bar{A}_{j, k}$.

Moreover, $\overline{\mathcal{A}}_{0}(\lambda)$ has $\tilde{h}$ eigenvectors not depending from $\lambda$ (shared with $\sum_{k=1}^{q} \lambda_{k}(t) \bar{A}_{i, k}$, and with $\sum_{k=1}^{q} \lambda_{k}(t) \bar{A}_{j, k}$ in the second case) that have 0 as associated eigenvalue. We call $\bar{Z} \in \mathbb{R}^{(n+1) \times \bar{h}}$ the full-rank matrix of these eigenvectors $\left(\overline{\mathcal{A}}_{0}(\lambda) \bar{Z}=\sum_{k=1}^{q} \lambda_{k}(t) \bar{A}_{j, k} \bar{Z}=\sum_{k=1}^{q} \lambda_{k}(t) \bar{A}_{i, k} \bar{Z}=0\right.$; this implies that the equilibrium set can be larger than the origin alone, i.e. $\left.E_{\text {eq }} \supseteq\{0\}\right)$. We then define $\bar{\Pi} \in \mathbb{R}^{(n+1) \times((n+1)-\bar{h})}$ the orthogonal complement of $\bar{Z}$, with $\bar{\Pi}^{T} \bar{\Pi}=I$.

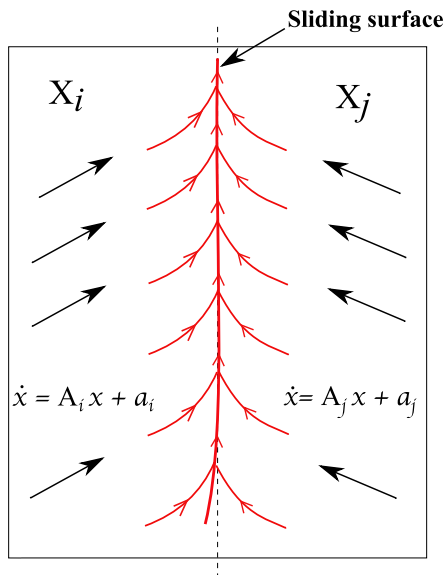

Fig. 6. Sliding modes.

The objective is to find a set of conditions ensuring the exponential convergence of the system state $x(t)$ to the set of equilibrium points $E_{e q}$, notwithstanding the presence of sliding modes on the boundaries and parametric variations in the model. Basically we have to upgrade the methods in [19], [15] in order to cope with sliding modes, equilibrium sets and parametric variations; we will use a Lyapunov function of degree higher than 2 (as done in [2]), i.e. a piecewisepolynomial Lyapunov function, which is less conservative than the classical piecewise-quadratic Lyapunov functions. Such functions are given by the expression

$$
V(x)=V_{i}(x) \text { for } x \in X_{i}, i \in \mathcal{I}
$$

such that $V_{i}(x)$ is a polynomial in $n$ variables with degree $2 m$ given by:

$$
V_{i}(x)=\chi(x)^{T} P_{i} \chi(x)
$$

where $P_{i}=P_{i}^{T} \in \mathbb{R}^{\rho \times \rho}$, and $\chi(x) \in \mathbb{R}^{\rho \times 1}$ is the vector of all the monomials of degree less than or equal to $m$ that can be made from the elements of $x ; \rho=\left(\begin{array}{c}m+n \\ m\end{array}\right)$. For example,

$$
\begin{aligned}
\chi(x)= & {\left[x_{1}, x_{2}, \ldots, x_{n}, x_{1} x_{2}, x_{1} x_{3}, \ldots, x_{1} x_{n}, x_{2} x_{3}, \ldots\right.} \\
& \left.\ldots, x_{2} x_{n}, \ldots, x_{n-1} x_{n}, \ldots, x_{1}^{m}, x_{2}^{m}, \ldots, x_{n}^{m}, 1\right]^{T} .
\end{aligned}
$$

As seen in [24], the choice of $P_{i}$ is not unique for a given polynomial $V_{i}(x)$; there exists a number $\iota$ of linearly inde- 
pendent matrices $Q_{\nu}=Q_{\nu}^{T} \in \mathbb{R}^{\rho \times \rho}$ (defined up to a scalar factor), for which

$$
\chi(x)^{T} Q_{\nu} \chi(x)=0 \text { for } \nu=1, \ldots, \iota
$$

with

$$
\iota=\frac{1}{2}\left(\left(\begin{array}{c}
m+n \\
m
\end{array}\right)^{2}+\left(\begin{array}{c}
m+n \\
m
\end{array}\right)\right)-\left(\begin{array}{c}
n+2 m \\
2 m
\end{array}\right) .
$$

This implies that $V_{i}(x)=\chi(x)^{T} P_{i} \chi(x)=\chi(x)^{T}\left(P_{i}+\right.$ $\left.\sum_{\nu=1}^{\iota} Q_{\nu} \tau(\nu)\right) \chi(x)$ for any real scalars $\tau(\nu)$. This is due to the fact that some monomials in $V_{i}$ can be obtained as products of different elements in $\chi$, e.g. $x_{1}^{2}=x_{1} \cdot x_{1}=1 \cdot x_{1}^{2}=x_{1}^{2} \cdot 1$.

Following the procedure of the "power transformation" [32], [30], we can obtain the dynamics of $\chi(x(t))=\chi(t)$, with which we will be able to compute the time derivative of the Lyapunov function. Namely, the uncertain dynamics will still be PWA, i.e. we will have

$\dot{\chi}(t)=\sum_{k=1}^{q_{i}} \lambda_{k}(t) \tilde{A}_{i, k} \chi(t), \forall k \in\{1, \ldots, q\}$, for $\chi \in \tilde{X}_{i}, i \in \mathcal{I}$

and we can construct a new description of the cells $\tilde{X}_{i}$, with matrices $\tilde{E}_{i} \in \mathbb{R}^{\tilde{l}_{i} \times \rho}$ such as

$$
\left\{\begin{array}{l}
x \in X_{i} \Rightarrow \chi \in \tilde{X}_{i} \\
\chi \in \tilde{X}_{i} \Rightarrow \tilde{E}_{i} \chi \geq 0
\end{array}\right.
$$

and defining the boundaries, with $\tilde{F}_{i j} \in \mathbb{R}^{\tilde{r}_{i j} \times \rho}$, as

$$
\forall(i, j) \in \Gamma, \tilde{X}_{i} \cap \tilde{X}_{j} \subseteq\left\{\chi \mid \tilde{F}_{i j} \chi=0\right\}
$$

Notice that, counterintuitively, the power transformation leaves $\lambda$ as linear terms in the dynamic equations for $\chi$. We define $\tilde{A}_{i}(\lambda)=\sum_{k=1}^{q} \lambda_{k} \tilde{A}_{i, k}$. Assumptions 1, Assumption 2 and Assumption 3 are also still holding for the new system in (17):

1) Sliding modes may occur, with the following dynamics:

$$
\begin{gathered}
\dot{\chi}(t)=\left(\theta(\lambda) \sum_{k=1}^{q} \lambda_{k}(t) \tilde{A}_{i, k}+(1-\theta(\lambda)) \sum_{k=1}^{q} \lambda_{k}(t) \tilde{A}_{j, k}\right) \chi(t), \\
0 \leq \theta(\lambda) \leq 1
\end{gathered}
$$

2) There exists an equilibrium set $\tilde{E}_{e q}$ such that $\chi \in \tilde{E}_{e q} \Leftrightarrow$ $\dot{\chi}=0 ; \chi \in \tilde{E}_{e q} \Leftrightarrow x \in E_{e q} ; \tilde{E}_{e q} \subset \bigcup_{i \in \mathcal{I}_{0}} \tilde{X}_{i}$

3) Again we distinguish two cases:

- if the origin is in the interior of a cell $\tilde{X}_{i} ; \tilde{A}_{i, k}(\lambda)$ does not have any constant terms for all $\lambda ; \tilde{\mathcal{A}}_{0}(\lambda)=$ $\sum_{k=1}^{q} \lambda_{k}(t) \tilde{A}_{i, k}=\tilde{A}_{i, k}(\lambda)$;

- if the origin is in the boundary between cells $\tilde{X}_{i}$ and $\tilde{X}_{j}$ : there exists a $\tilde{\theta}(\lambda), 0 \leq \tilde{\theta}(\lambda) \leq$ 1 , for which the constant terms disappear; we define $\tilde{\mathcal{A}}_{0}(\lambda)=\tilde{\theta}(\lambda) \quad \sum_{k=1}^{q} \lambda_{k}(t) \tilde{A}_{i, k}+(1-$ $\tilde{\theta}(\lambda)) \quad \sum_{k=1}^{q} \lambda_{k}(t) \tilde{A}_{j, k}=\theta(\lambda) \tilde{A}_{i}(\lambda)+(1-$ $\tilde{\theta}(\lambda)) \tilde{A}_{j}(\lambda)$.

Identically, $\tilde{\mathcal{A}}_{0}(\lambda)$ has $\tilde{h}$ eigenvectors not depending from $\lambda$ (shared with $\sum_{k=1}^{q} \lambda_{k}(t) \tilde{A}_{i, k}$ and with $\sum_{k=1}^{q} \lambda_{k}(t) \tilde{A}_{j, k}$ in the second case) that have 0 as associated eigenvalue. We can also call $\tilde{Z} \in \mathbb{R}^{\rho \times \tilde{h}}$ the full-rank matrix of these eigenvectors $\left(\tilde{\mathcal{A}}_{0}(\lambda) \tilde{Z}=\right.$ $\sum_{k=1}^{q} \lambda_{k}(t) \tilde{A}_{j, k} \tilde{Z}=\sum_{k=1}^{q} \lambda_{k}(t) \tilde{A}_{i, k} \tilde{Z}=0$ ); we define $\tilde{\Pi} \in \mathbb{R}^{\rho \times(\rho-\tilde{h})}$ as the orthogonal complement of $\tilde{Z}$, with $\tilde{\Pi}^{T} \tilde{\Pi}=I$.

$V(\chi)$ in (12) and (13) is a candidate Lyapunov function for the system in (8). In order to ensure its continuity on the boundary between two cells $\tilde{X}_{i}$ and $\tilde{X}_{j},(i, j) \in \Gamma$, the following condition has to be satisfied:

$$
V_{i}(\chi)=V_{j}(\chi) \forall \chi(x) \in \tilde{X}_{i} \cap \tilde{X}_{j} .
$$

As a direct consequence of LaSalle's theorem ([21], [31]), the conditions that the candidate time-invariant Lyapunov function must satisfy in order to prove the convergence to the equilibrium set are in the following lemma.

Lemma 3: For the system in (8), the convergence of $x$ to $E_{\text {eq }}$ (which coincides with the convergence of $\chi$ in (17) to $\tilde{E}_{e q}$ ) for $t>0, t \rightarrow+\infty$ is assured under the following conditions.

1) The Lyapunov function is continuous, i.e. it satisfies (21);

2) The Lyapunov function is positive outside $\tilde{E}_{e q}$,

$$
V_{i}(\chi(x))>0 \text { for } \chi(x) \in \tilde{X}_{i} / \tilde{E}_{e q}, i \in \mathcal{I} ;
$$

3) The derivative of the Lyapunov function is negative outside $\tilde{E}_{e q}$, i.e.

$$
\dot{V}_{i}(\chi(x))<0 \text { for } \chi(x) \in \tilde{X}_{i} / \tilde{E}_{e q}, i \in \mathcal{I} ;
$$

even in the case of sliding-mode dynamics;

4) The derivative of the Lyapunov function is null in $\tilde{E}_{e q}$, i.e.

$$
\dot{V}_{i}(\chi(x))=0 \text { for } \chi(x) \in \tilde{E}_{e q} \cap \tilde{X}_{i}, i \in \mathcal{I}_{0} .
$$

We will also try and evaluate the speed of the exponential convergence of the system trajectories to the equilibrium set $E_{e q}$. For this purpose, we can define the distance $d$ from a state $x$ to any point in $E_{e q}$ as

$$
d\left(x, E_{e q}\right)=\inf _{x_{e q} \in E_{e q}}\left\|x-x_{e q}\right\| .
$$

We can then introduce the following definition.

Definition 4 (Decay rate): We call "decay rate" the largest positive number $\alpha$ such that for any initial condition $x_{0}$ :

$$
\lim _{t \rightarrow \infty} e^{\alpha t} d\left(x(t), E_{e q}\right)=0 .
$$

Corollary 5: The decay rate of system in (8) is larger then $\alpha$ if the conditions in (23) are replaced by

$$
\left\{\begin{array}{l}
\dot{V}_{i}\left(\tilde{\Pi} \tilde{\Pi}^{T} \chi(x)\right)<-2 \alpha V_{i}\left(\tilde{\Pi} \tilde{\Pi}^{T} \chi(x)\right) \text { for } \chi(x) \in \tilde{X}_{i} / \tilde{E}_{e q}, i \in \mathcal{I}_{0} \\
\dot{V}_{i}(\chi(x))<-2 \alpha V_{i}(\chi(x)) \text { for } \chi(x) \in \tilde{X}_{i} i \in \mathcal{I}_{1} .
\end{array}\right.
$$

\section{MAIN RESUlT}

Given the previous considerations and using the S-procedure [4], we can arrive at our main theoretical result, which reveals that a Lyapunov function can be computed through an LMI optimization.

Theorem 6: Let us assume that the system in (8) satisfies Assumption 1, Assumption 2 and Assumption 3. Let $\tilde{A}_{i, k}$, 
$\forall k \in\{1, \cdots, q\}$ be the uncertain matrices describing the dynamics of the vector $\chi$ of degree $m$ deriving from $x$, as in (17), with $\tilde{X}_{i}, \tilde{E}_{i}, \tilde{F}_{i j}, \tilde{Z}_{i}$, etc. as previously defined.

If there exist

- $P_{i}=P_{i}^{T} \in \mathbb{R}^{\rho \times \rho}$ for $i \in \mathcal{I}$;

- $U_{i, k}=U_{i, k}^{T}, W_{i}=W_{i}^{T} \in \mathbb{R}^{\tilde{l}_{i} \times \tilde{l}_{i}}$ and $\mathcal{T}_{i, k}, \mathcal{T}_{i}^{\prime}, \in \mathbb{R}^{\tilde{l}_{i}}$; all with non-negative entries for $i \in \mathcal{I}, k=1, \ldots, q$;

- $U_{i j, k}, U_{i j, k}^{\prime} \in \mathbb{R}^{\tilde{l}_{i} \times \tilde{l}_{i}}, \mathcal{T}_{i j, k}, \mathcal{T}_{i j, k}^{\prime} \in \mathbb{R}^{\tilde{l}_{i}}$, all with nonnegative entries for $i, j \mid(i, j) \in \Gamma, k=1, \ldots, q$;

- $L_{i j} \in \mathbb{R}^{\rho \times \tilde{r}_{i j}}$ for $i, j \mid(i, j) \in \Gamma$;

- $\tau_{i}(\nu), \tau_{i}^{\prime}(\nu), \tau_{i, k}^{\prime \prime}(\nu), \tau_{i j, k}(\nu), \tau_{i j}(\nu) \in \mathbb{R}$, for $i, j \in \mathcal{I}$, $\nu=1, \ldots, \iota, k=1, \ldots, q$

such that

$$
\begin{gathered}
P_{j}=P_{i}+\tilde{F}_{i j}^{T} L_{i j}^{T}+L_{i j} \tilde{F}_{i j}+H\left(\tau_{i j}\right) \text { for }(i, j) \in \Gamma \\
\left\{\begin{array}{l}
\tilde{\Pi}^{T} \tilde{A}_{i, k}^{T} P_{i} \tilde{Z}=0 \\
\tilde{E}_{i} \tilde{Z}=0
\end{array} \text { for } i \in \mathcal{I}_{0}, k=1, \ldots, q\right.
\end{gathered}
$$

and it holds that

$$
\left\{\begin{array}{l}
\tilde{\Pi}^{T}\left(P_{i}+H\left(\tau_{i}^{\prime}\right)-N_{i}\left(\mathcal{T}_{i}^{\prime}\right)-\tilde{E}_{i}^{T} W_{i} \tilde{E}_{i}\right) \tilde{\Pi} \succ 0 \\
\tilde{\Pi}^{T}\left(\tilde{A}_{i, k}^{T} P_{i}+P_{i} \tilde{A}_{i, k}+H\left(\tau_{i, k}^{\prime \prime}\right)+N_{i}\left(\mathcal{T}_{i, k}\right)+\tilde{E}_{i}^{T} U_{i, k} \tilde{E}_{i}\right) \tilde{\Pi} \\
\quad+2 \alpha \tilde{\Pi}^{T} P_{i} \tilde{\Pi} \prec 0
\end{array}\right.
$$$$
\text { for } i \in \mathcal{I}_{0}, k=1, \ldots, q
$$$$
\left\{\begin{array}{l}
P_{i}+H\left(\tau_{i}^{\prime}\right)-N_{i}\left(\mathcal{T}_{i}^{\prime}\right)-\tilde{E}_{i}^{T} W_{i} \tilde{E}_{i} \succ 0 \\
\tilde{A}_{i, k}^{T} P_{i}+P_{i} \tilde{A}_{i, k}+H\left(\tau_{i, k}^{\prime \prime}\right)+N_{i}\left(\mathcal{T}_{i, k}\right)+\tilde{E}_{i}^{T} U_{i, k} \tilde{E}_{i} \\
+2 \alpha P_{i} \prec 0
\end{array}\right.
$$$$
\text { for } i \in \mathcal{I}_{1}, k=1, \ldots, q
$$

$$
\left\{\begin{array}{l}
\tilde{\Pi}^{T}\left(\tilde{A}_{i, k}^{T} P_{j}+P_{j} \tilde{A}_{i, k}+H\left(\tau_{i j, k}\right)+N_{i}\left(\mathcal{T}_{i j, k}\right)+N_{j}\left(\mathcal{T}_{i j, k}^{\prime}\right)\right. \\
\left.\quad+\tilde{E}_{i}^{T} U_{i j, k} \tilde{E}_{i}+\tilde{E}_{j}^{T} U_{i j, k}^{\prime} \tilde{E}_{j}\right) \tilde{\Pi}+2 \alpha \tilde{\Pi}^{T} P_{j} \tilde{\Pi} \prec 0 \\
\tilde{\Pi}^{T}\left(\tilde{A}_{j, k}^{T} P_{i}+P_{i} \tilde{A}_{j, k}+H\left(\tau_{j i, k}\right)+N_{i}\left(\mathcal{T}_{j i, k}\right)+N_{j}\left(\mathcal{T}_{j i, k}^{\prime}\right)\right. \\
\left.\quad+\tilde{E}_{j}^{T} U_{j i, k} \tilde{E}_{j}+\tilde{E}_{i}^{T} U_{j i, k}^{\prime} \tilde{E}_{i}\right) \tilde{\Pi}+2 \alpha \tilde{\Pi}^{T} P_{i} \tilde{\Pi} \prec 0 \\
\tilde{\Pi}^{T} \tilde{A}_{i, k}^{T} P_{j} \tilde{Z}=0, \tilde{\Pi}^{T} \tilde{A}_{j, k}^{T} P_{i} \tilde{Z}=0
\end{array}\right.
$$$$
\text { for }(i, j) \in \Gamma, i, j, \in \mathcal{I}_{0}, k=1, \ldots, q
$$$$
\left\{\begin{array}{l}
\tilde{A}_{i, k}^{T} P_{j}+P_{j} \tilde{A}_{i, k}+H\left(\tau_{i j, k}\right)+N_{i}\left(\mathcal{T}_{i j, k}\right)+N_{j}\left(\mathcal{T}_{i j, k}^{\prime}\right) \\
+\tilde{E}_{i}^{T} U_{i j, k} \tilde{E}_{i}+\tilde{E}_{j}^{T} U_{i j, k}^{\prime} \tilde{E}_{j}+2 \alpha P_{j} \prec 0 \\
\tilde{A}_{j, k}^{T} P_{i}+P_{i} \tilde{A}_{j, k}+H\left(\tau_{j i, k}\right)+N_{i}\left(\mathcal{T}_{j i, k}\right)+N_{j}\left(\mathcal{T}_{j i, k}^{\prime}\right) \\
+\tilde{E}_{j}^{T} U_{j i, k} \tilde{E}_{j}+\tilde{E}_{i}^{T} U_{j i, k}^{\prime} \tilde{E}_{i}+2 \alpha P_{i} \prec 0 \\
\quad \text { for }(i, j) \in \Gamma, i \notin \mathcal{I}_{0} \bigvee j \notin \mathcal{I}_{0}, k=1, \ldots, q
\end{array}\right.
$$

with

$$
N_{i}(\mathcal{T})=\left[\begin{array}{c}
0 \\
\mathcal{T}^{T} \tilde{E}_{i}
\end{array}\right]+\left[\begin{array}{ll}
0 & \tilde{E}_{i}^{T} \mathcal{T}
\end{array}\right]
$$

and

$$
H(\tau)=\sum_{\nu=1}^{\iota} \tau(\nu) Q_{\nu}
$$

then the trajectories $x(t)$ of the system (8) converge exponentially to the equilibrium set $E_{e q}$, with a decay rate larger than $\alpha$, with the Lyapunov function (12).

Proof: Consider the Lyapunov function candidate $V(\chi(x))$ defined by (12).
To prove (26), we use the same approach as in [15]. So, for $\chi(x) \in \tilde{X}_{i} \cap \tilde{X}_{j}$, we have $\tilde{F}_{i j} \chi(x)=0$; replacing this into (26), multiplying on the right by $\chi(x)$ and on the left by $\chi(x)^{T}$, and remembering that $\chi(x)^{T} H(\tau) \chi(x)=0$ for any $\tau$, we get $\chi(x)^{T} P_{j} \chi(x)=\chi(x)^{T} P_{i} \chi(x)$, that is (21), i.e. (26) implies continuity of the Lyapunov function on the boundaries. Let us now consider the inequalites; all of the inequalities involving $\tilde{A}_{i, k}$ are present for all the values of $k$, assuring that such equalities will also hold for $\tilde{A}_{i}(\lambda)$ for any valid $\lambda$. For example, if $\tilde{A}_{i, k}^{T} P_{i}+P_{i} \tilde{A}_{i, k}+H\left(\tau_{i, k}^{\prime \prime}\right)+N_{i}\left(\mathcal{T}_{i, k}\right)+\tilde{E}_{i}^{T} U_{i, k} \tilde{E}_{i}+$ $2 \alpha P_{i} \prec 0$ holds for all $k$, then $\tilde{A}_{i}(\lambda)^{T} P_{i}+P_{i} \tilde{A}_{i}(\lambda)+H\left(\tau_{i}^{\prime \prime}\right)+$ $N_{i}\left(\mathcal{T}_{i}\right)+\tilde{E}_{i}^{T} U_{i} \tilde{E}_{i}+2 \alpha P_{i} \prec 0$ holds for all $\lambda$, with $\tau_{i}^{\prime \prime}, \mathcal{T}_{i}, U_{i}$ appropriately chosen. We will consider this as granted for the follow-up of the proof, for all of the inequalites.

Let us now consider (27) and (28). Based on Assumption 3, we can always write a decomposition for $\chi(x)$, of the kind $\chi(x)=\tilde{Z} \xi+\tilde{\Pi} \zeta$, with $\chi(x) \in \tilde{X}_{i}, i \in \mathcal{I}_{0}$. As $\tilde{\mathcal{A}}_{0}(\lambda) \tilde{Z}=0$ for $\chi(x) \in \tilde{E}_{e q}$ (i.e. $\chi(x)=\tilde{Z} \xi$ ), then the first in (27) implies (24). For $\chi(x) \notin \tilde{E}_{e q}$ instead, when the active dynamics is $\tilde{A}_{i}(\lambda)$, with $i \in \mathcal{I}_{0}$, we have $\dot{V}(\chi(x))=(\tilde{Z} \xi+\tilde{\Pi} \zeta)^{T}\left(\tilde{A}_{i}(\lambda)^{T} P_{i}+P_{i} \tilde{A}_{i}(\lambda)\right)(\tilde{Z} \xi+\tilde{\Pi} \zeta)=$ $\zeta^{T} \tilde{\Pi}^{T}\left(\tilde{A}_{i}(\lambda)^{T} P_{i}+P_{i} \tilde{A}_{i}(\lambda)^{T}\right) \tilde{\Pi} \zeta$ thanks to (27) (notice that: $\left.\tilde{\Pi}^{T} \tilde{A}_{i}(\lambda)^{T} P_{i} \tilde{Z}=0\right)$. Notice also that the second in (27) implies that $\tilde{E}_{i} \chi \geq 0 \Rightarrow \tilde{E}_{i} \tilde{\Pi} \zeta \geq 0$. Then, thanks to the S-procedure, the second inequality in (28) implies $\dot{V}_{i}\left(\tilde{\Pi} \tilde{\Pi}^{T} \chi(x)\right)<-2 \alpha V_{i}\left(\tilde{\Pi} \tilde{\Pi}^{T} \chi(x)\right)$ for $\chi(x) \notin \tilde{E}_{e q}$, which is the first in (25), as we have $\zeta^{T} \tilde{\Pi}^{T} \tilde{E}_{i}^{T} U_{i, k} \tilde{E}_{i} \tilde{\Pi} \zeta \geq 0$ (true thanks to (18)) and (27)), $\zeta^{T} \tilde{\Pi}^{T} N_{i}\left(\mathcal{T}_{i, k}^{\prime}\right) \tilde{\Pi} \zeta \geq 0$ (true thanks to (18) and (27)) and $\chi(x)^{T} H\left(\tau_{i, k}^{\prime}\right) \chi(x)=0$ (true thanks to (15)). In a similar way, the first inequality in (28) naturally implies the inequality (22) for the cells with $i \in \mathcal{I}_{0}$.

Subsequently, again thanks to the S-procedure, the first inequality in (29) implies that $\chi(x)^{T} P_{i} \chi(x)>0$ when $\chi(x)^{T} \tilde{E}_{i}^{T} W_{i} \tilde{E}_{i} \chi(x) \geq 0$ (true thanks to (18) if $\left.\chi(x) \in \tilde{X}_{i}\right), \chi(x)^{T} N_{i}\left(\mathcal{T}_{i}^{\prime}\right) \chi(x) \geq 0$ (true thanks to (18)) $\chi(x)^{T} H\left(\tau_{i}^{\prime}\right) \chi(x)=0$ (true thanks to (15)), which in turn implies that $V_{i}(\chi(x))>0$ for $\chi(x) \in \tilde{X}_{i}$ i.e. the inequality (22) for the cells with $i \in \mathcal{I}_{1}$. Similarly, the second inequality in (29) ensures the second inequality in (25).

The conditions (30) and (31) concern possible sliding modes on the boundary between two cells. Indeed, multiplying the second inequality in (31) by $\theta$ (omitting the dependency on $\lambda$ ) and the second in (28) by $1-\theta$ and summing, we get (thanks to the S-procedure, as seen above)

$$
\begin{gathered}
\chi(x)^{T}\left(\left(\theta \tilde{A}_{j}(\lambda)^{T}+(1-\theta) \tilde{A}_{i}(\lambda)^{T}\right) P_{i}+P_{i}\left(\theta \tilde{A}_{j}(\lambda)+\right.\right. \\
\left.\left.(1-\theta) \tilde{A}_{i}(\lambda)\right)+2 \alpha P_{i}\right) \chi(x)<0
\end{gathered}
$$

for any $\chi \in \tilde{X}_{i} \cap \tilde{X}_{j}$, which ensures that the derivative of $V_{i}$ is sufficiently negative for the given decay rate for any possible sliding mode dynamics. Similarly, one can get the complementary inequality

$$
\begin{gathered}
\chi(x)^{T}\left(\left(\theta \tilde{A}_{j}(\lambda)^{T}+(1-\theta) \tilde{A}_{i}(\lambda)^{T}\right) P_{j}+P_{j}\left(\theta \tilde{A}_{j}(\lambda)+\right.\right. \\
\left.\left.(1-\theta) \tilde{A}_{i}(\lambda)\right)+2 \alpha P_{j}\right) \chi(x)<0
\end{gathered}
$$

from the first in (31). Condition (30) concerns a sliding mode containing the equilibrium set, the inequalities can be proven with the same reasoning as for (31) and (28). 
So we have shown that the conditions required by the theorem imply (21), (22), (24) and (25), satisfying the hypotheses of Lemma 3, which proves the theorem statement.

The theorem involves a feasibility problem under linear matrix inequalities (LMIs) in (28), (29), (30) and (31), as well as linear matrix equalities (LMEs), (26) and (27), and the last in (30). Such LMEs can be resolved by an appropriate parameterization of the unknowns. In the case of $\alpha=0$, the theorem proves the simple asymptotic stability of the system in (8) with respect to $E_{e q}$. An interesting problem is then to find the largest $\alpha$ such that Theorem 6 is satisfied. This problem can be solved through a dichotomic search with respect to $\alpha$.

Remark 7: The first conditions in (28) and (29) can be interpreted as a "sum of squares" property (SOS) [17], [24], [10]. In fact we look for a positive definite polynomial $V_{i}(\chi)$ as a square product of a positive definite matrix times a vector of monomials $\chi$. The terms in $H(\tau)$ reduce the conservatism of the inequality.

Remark 8: Theorem 6 includes several previous results as special cases.

- In the case of no sliding modes, no uncertain parameters, for $m=1, E_{e q}=\{0\}, \mathcal{T}_{\bullet}=0$ and $\alpha=0$, we have Theorem 1 in [19].

- In the case of no sliding modes, no uncertain parameters, for $m=1, E_{e q}=\{0\}, U_{\bullet}=0$ and $\alpha=0$, we have the theorem in Section 5 of [15].

- In the case of no sliding modes, no uncertain parameters, for $m=1$, we have Theorem 9 in [1].

- With sliding modes allowed, no uncertain parameters, for $m \geq 1$ and $\alpha=0$, we have Theorem 3 in [2].

In this sense, Theorem 6 is an extension of these previous results; it is less conservative as it features a more general condition, and at the same times it allows the analysis of the convergence to equilibrium sets other than the origin alone even in the presence of sliding modes and parametric uncertainties.

\section{ApPlication to the PNEUMATIC ACtUATOR}

As shown in [1], and according to (4) and (5), the dynamical behavior of the pneumatic system described in Section II can then be cast into the form of a PWA system as in (8). Concerning the parameters of the test bench, we have $l=0.5 \mathrm{~m}, M=17 \mathrm{~kg}, F_{s}=38 \mathrm{~N}$, $S=7.27 \cdot 10^{-4} \mathrm{~m}^{2}, \varepsilon=0.1 \mathrm{~m} / \mathrm{s}, \varepsilon_{1}=0.005 \mathrm{~m}$ (which implies that the final positioning error is smaller in modulus than this value), $\varepsilon_{2}=0.01 \mathrm{~m} / \mathrm{s}$ and $\varepsilon_{3}=10^{-4} \mathrm{~m} / \mathrm{s}$. Through a pole placement, according to the system specifications, we have set $k_{y}=50 \mathrm{~s}^{-3}, k_{v}=71 \mathrm{~s}^{-2}, k_{a}=51.4 \mathrm{~s}^{-1}$, $k_{P}=10 \mathrm{~s}^{-1}$ and $k_{N}=10 \mathrm{~s}^{-1}$.

Theorem 6 can be applied in several different cases, according to the friction model chosen (saturation form or relay form), the type of problem under consideration (stability or performance study), and the value of $m$. We will present the results of the possible cases comparing the outcome of using piecewise-quadratic Lyapunov functions $(m=1)$ and piecewise-quartic ones $(m=2)$. We study the asymptotic and the exponential stability of the equilibrium set $E_{e q}=$ $\left[\begin{array}{llll}x_{1}^{e} & 0 & 0 & 0\end{array}\right]^{T}$, where $x_{1}^{e}$ represents a steady position error between $-\varepsilon_{1}$ and $\varepsilon_{1}$. Finding a valid Lyapunov function is equivalent to proving the closed-loop stability of the pneumatic actuator (for any possible initial condition, including any possible switching sequence).

\section{A. Friction in saturation form}

We begin our study choosing friction in saturation form as in (3), which presents no sliding modes on the boundaries. For this model, the results of the theorem applications are presented as follows.

\section{- Stability study}

As we study the convergence of the state to $E_{e q}$, we consider a static setpoint, i.e. $y_{d}$ constant and $v_{d}=0$, $a_{d}=0$ (which implies $e_{v}=v, e_{a}=a$ ).

The switching criteria divide the state-space into seven cells (see Figure 7): a central cell $X_{0}$, for which $0 \in$ $X_{0}$, and six external cells $X_{1}, X_{2}, X_{3}, X_{4}, X_{5}$ and $X_{6}$. Certain couples of cells are symmetric to each other with respect to the origin: $X_{1}$ and $X_{2}, X_{3}$ and $X_{4}$, and $X_{5}$ and $X_{6}$. We can use this property by imposing conditions only for a single cell of each couple, and by symmetry these conditions will necessarily be verified for the other ones. So, we need to impose the conditions of Theorem 6 only for the central cell $(i=0)$ and for $i=1,3,5$. First we consider that there is no variation on friction; so $k=1, \lambda_{1}=1$.

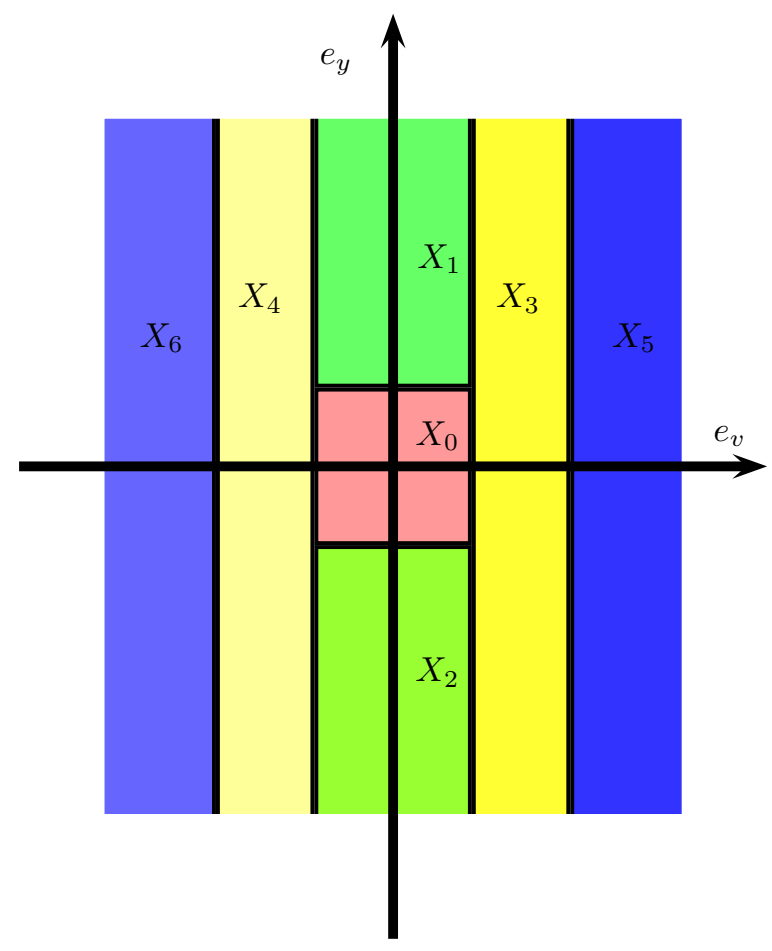

Fig. 7. The cells $X_{i}$ in the case of friction in saturation form, intersection with $\left(e_{v}, e_{y}\right)$ plane.

1) Piecewise quadratic Lyapunov function ( $m=1$ ) 
By applying Theorem 6 for $m=1$, the lower bound value on the decay rate found is: $\alpha=0.0897$.

Figure 8 presents the 2-dimensional level curves of the obtained Lyapunov function in the $\left(e_{v}, e_{y}\right)$ plane. We can clearly see that the level curves do not have a simple ellipsoidal shape; this suggests that a simple common quadratic Lyapunov function (i.e., the same matrix $P$ for all the cells) could be not sufficient to obtain such shapes. In fact, we have run our test also in order to look for a common quadratic Lyapunov function, and the test failed to find any. This justifies the effort in finding less conservative conditions as the ones of Theorem 6 .

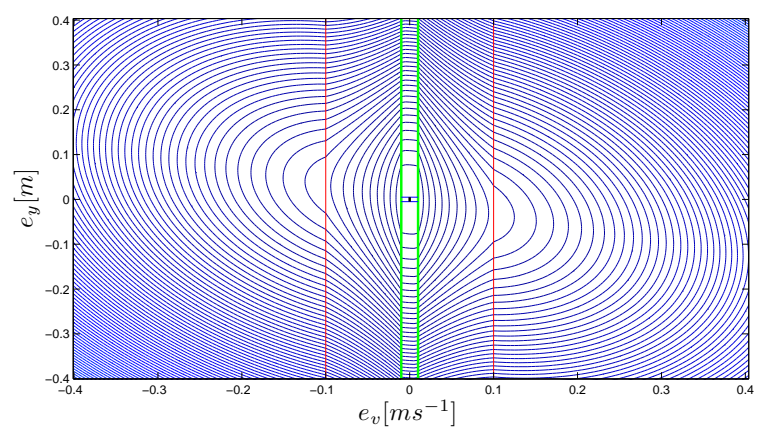

Fig. 8. Lyapunov function level curves, stability study, friction in saturation form, $m=1$.

2) Piecewise polynomial Lyapunov function ( $m=2$ ) By applying Theorem 6 for $m=2$, the lower bound value on the decay rate found is: $\alpha=0.4751$; which is larger than the one obtained in the case of $m=1$. This result shows the effectiveness of using a piecewise-polynomial Lyapunov function $(m=2)$ compared to the piecewise-quadratic one $(m=1)$. Figure 9 presents the 2-dimensional level curves of the obtained Lyapunov function in the $\left(e_{v}, e_{y}\right)$ plane

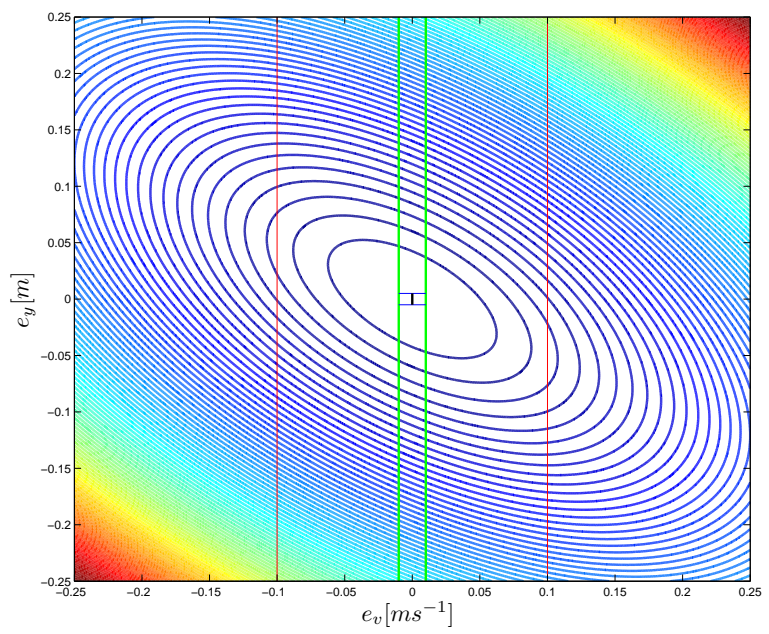

Fig. 9. Lyapunov function level curves, stability study, friction in saturation form, $m=2$.
On the other hand, we have also verified that for $m=2$ it is possible to find a common polynomial Lyapunov function, thanks to the lower conservatism with respect to a common quadratic one.

\section{- Robust stability}

For the robust stability study, we have modified the chosen model of friction by inserting a range of variation of the parameter $F_{s}$ (see for example Figure 10).

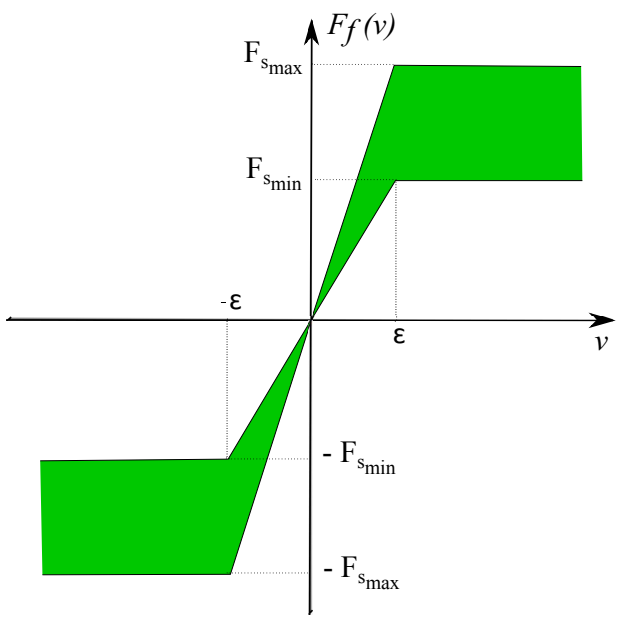

Fig. 10. Friction variation for the saturation case.

In our case study, we took a variation between $F_{s_{\max }}=$ $40 \mathrm{~N}$ and $F_{s_{\text {min }}}=20 \mathrm{~N}$. In this case, the friction model is polytopic with $k=1,2$, and can be presented according to (7) as:

$$
\left\{\begin{array}{l}
F_{s}(\lambda)=\lambda_{1} F_{s_{\max }}+\lambda_{2} F_{s_{\min }} \\
\lambda_{1}+\lambda_{2}=1
\end{array}\right.
$$

In this way, the model of the system is PWA with polytopic uncertainties.

1) Piecewise quadratic Lyapunov function $(m=1)$ By applying Theorem 6 for $m=1$, the lower bound value on the decay rate found is: $\alpha=0.0671$. The level curves of the obtained Lyapunov function are presented in the Figure 11.

2) Piecewise polynomial Lyapunov function $(m=2)$ By applying Theorem 6 for $m=2$, the lower bound value on the decay rate found is: $\alpha=0.4750$. For this case, the level curves of the Lyapunov function are pretty much the same compared to the case without variations.

For both cases, the stability was proven despite variations in friction, with a slight reduction of the decay rate.

- Performance study

We study the trajectory tracking by taking specified desired trajectories (see Figure 12), i.e in this case $y_{d}$ variable and $v_{d} \neq 0, a_{d} \neq 0$.

The cells are divided with respect to desired velocity switching criteria resulting into 9 cells (not shown for brevity).

1) Piecewise quadratic Lyapunov function ( $m=1)$ 


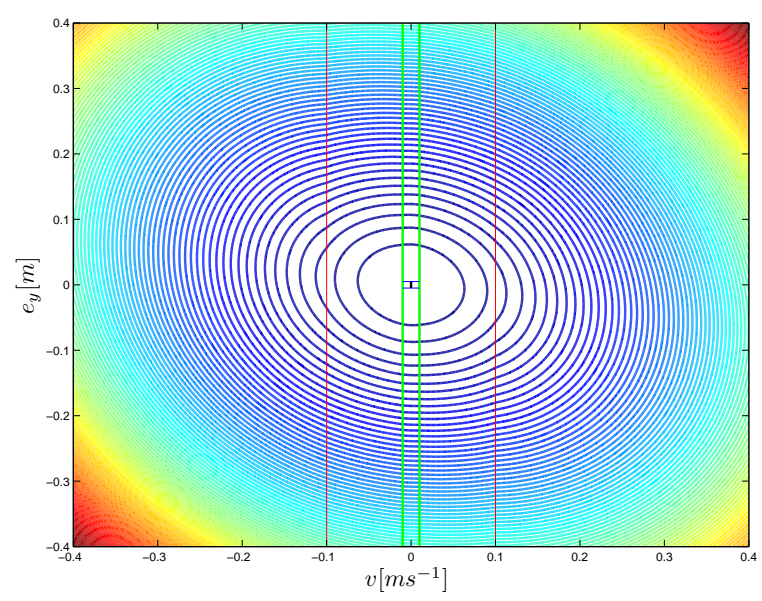

Fig. 11. Lyapunov function level curves, robust stability study, friction in saturation form, $m=1$.

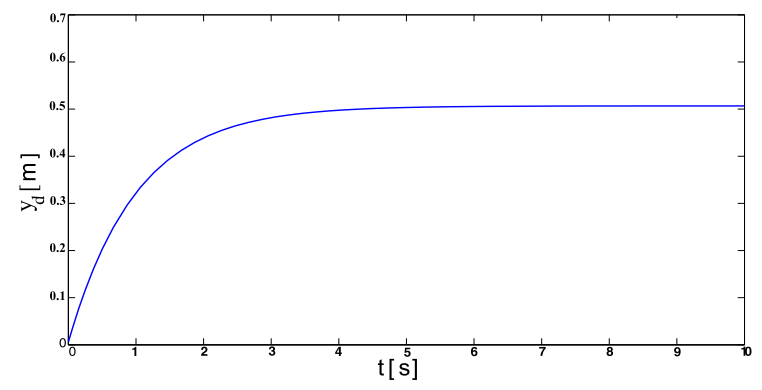

Fig. 12. Desired position $y_{d}$

By applying Theorem 6 for $m=1$ and $\alpha=0$, the asymptotic stability has been verified with a level curve of the obtained Lyapunov function presented in Figure 13 in the $\left(e_{y}, v, v_{d}\right)$ space. Again, we can point out that the shape is quite different from a simple ellipsoid, which makes it impossible to obtain by means of a common quadratic Lyapunov function.

2) Piecewise polynomial Lyapunov function $(m=2)$ The asymptotic stability has been also verified for $m=2$ and a level curve of the obtained Lyapunov function is presented in Figure 14 in the $\left(e_{y}, v, v_{d}\right)$ space.

\section{B. Friction in relay form}

The second friction model chosen for our study is in the relay form, as in (2), for which we study all the possible cases as done for the other friction model.

The switching of the system (due to the friction model and due to the control law) divides the state-space into a set of several cells; 8 cells considering only two switching criteria (see Figure 15), or 12 cells with respect to desired velocity switching criteria (not shown for brevity; more details on the PWA formulation of the model can be found in [2]).

The discontinuity of the friction model on $v=0$ can generate a sliding mode on the corresponding boundary, which

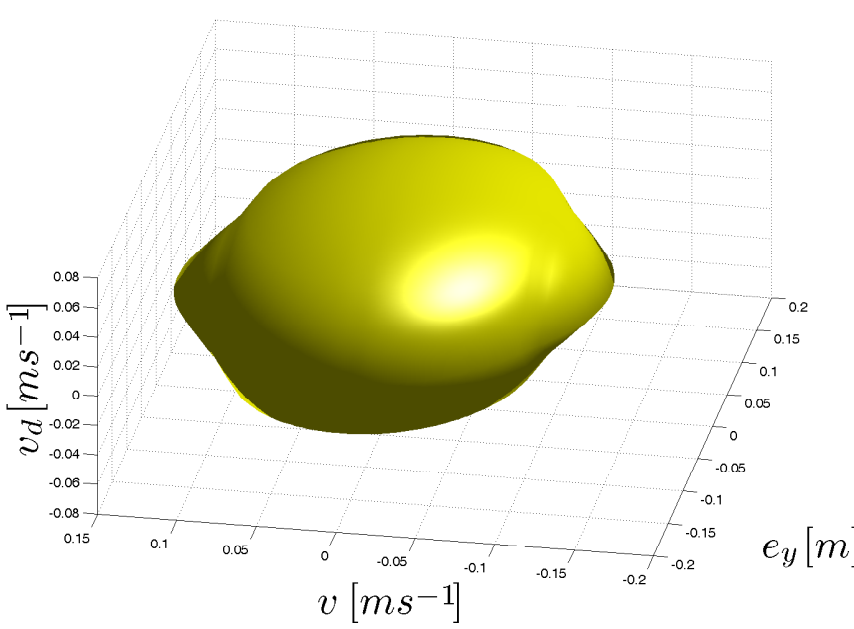

Fig. 13. A Lyapunov function level curve, performance study, friction in saturation form, $m=1$.

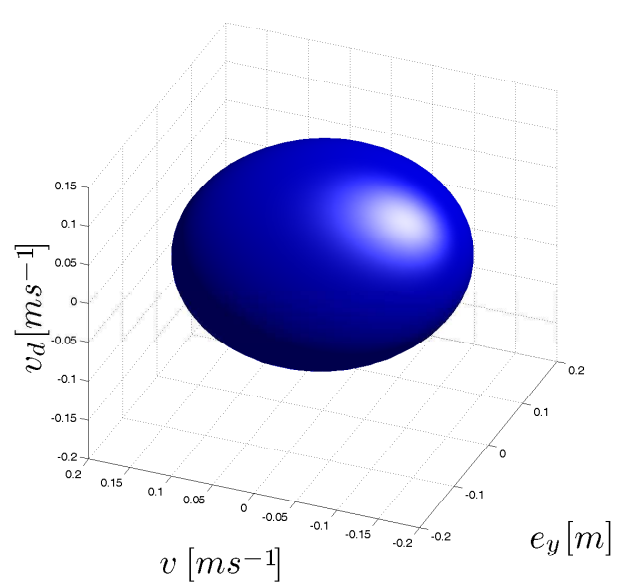

Fig. 14. A Lyapunov function level curve, performance study, friction in saturation form, $m=2$.

we need to take into account while proving stability. All the results of the theorem application concerning this friction model are presented in Table II.

To suggest an idea of the computations involved, we provide a brief discussion only for the performance study (trajectory tracking). In this case, the piecewise-polynomial function is of degree $2 m=4$, which yields a vector $\chi(x)$ of the extended state, according to (14), in the following form (remember that $x=\left[x_{1}, x_{2}, x_{3}, x_{4}, x_{5}, x_{6}\right]^{T}$, where $x_{5}, x_{6}$ are states associated to the reference trajectory): $\chi(x)=\left[x_{1}, x_{2}, x_{3}\right.$, $x_{4}, x_{5}, x_{6}, x_{1} x_{2}, x_{1} x_{3}, x_{1} x_{4}, x_{1} x_{5}, x_{1} x_{6}, x_{2} x_{3}, x_{2} x_{4}, x_{2} x_{5}$, $x_{2} x_{6}, x_{3} x_{4}, x_{3} x_{5}, x_{3} x_{6}, x_{4} x_{5}, x_{4} x_{6}, x_{5} x_{6}, x_{1}^{2}, x_{2}^{2}, x_{3}^{2}, x_{4}^{2}$, $\left.x_{5}^{2}, x_{6}^{2}, 1\right]^{T}$. The level curves of the piecewise-polynomial Lyapunov function found have been plotted in Figure 16 in the $\left(v, e_{y}\right)$ plane and in Figure 17 in the $\left(e_{y}, v, v_{d}\right)$ space. We can see that the level curves do not have a simple ellipsoidal shape; this implies again that a simple common quadratic Lyapunov function is not sufficient to obtain such shapes. Moreover, we have verified that the test fails for the case of $m=1$, which 


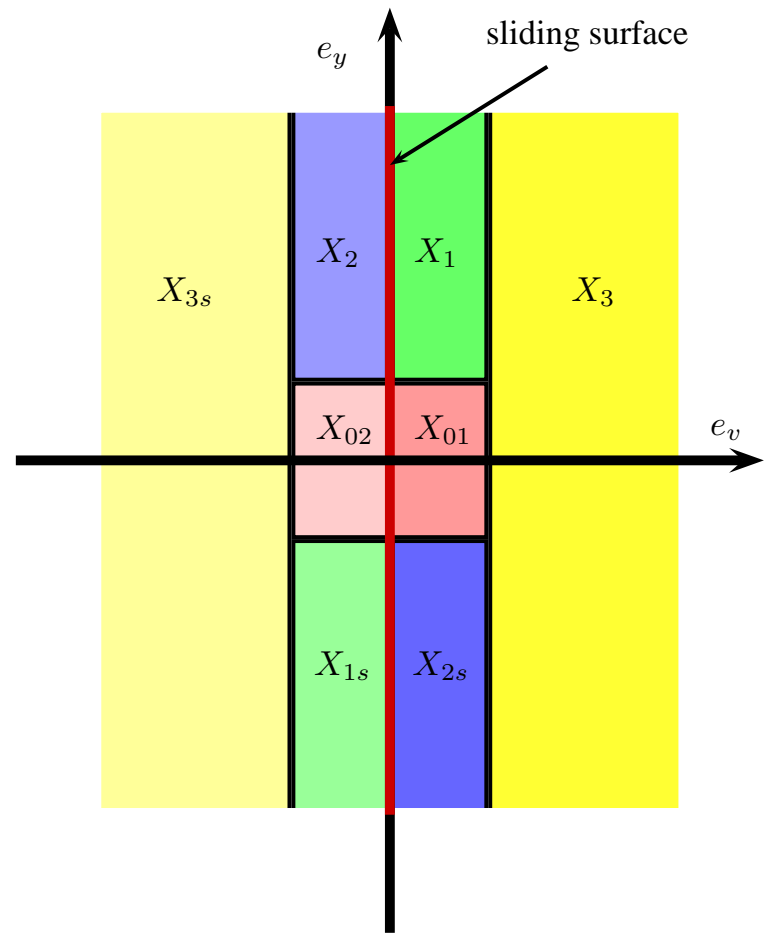

Fig. 15. The cells $X_{i}$ in the case of friction in relay form, intersection with $\left(e_{v}, e_{y}\right)$ plane.

justifies the need of a piecewise-polynomial function instead of a piecewise-quadratic one.

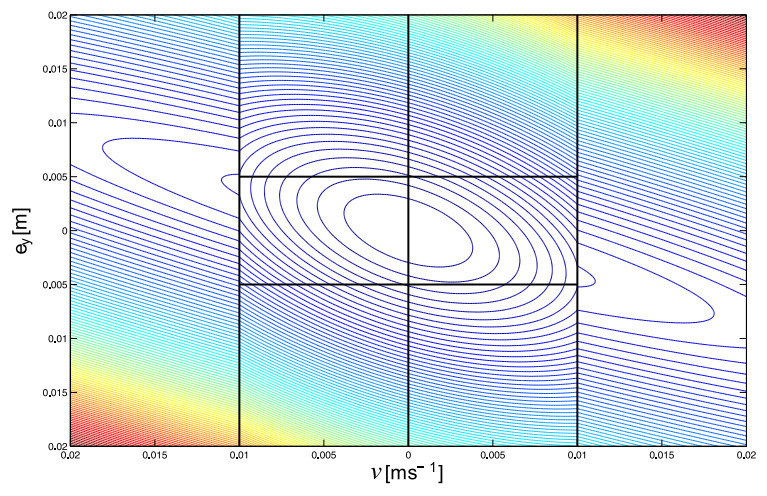

Fig. 16. Lyapunov function level curves, friction in relay form, performance study, $m=2$.

\section{Summary}

Table II contains the results for all cases of the theorem application to our electropneumatic system; namely stability, robust stability, performance and robust performance under the two models of friction and two forms of Lyapunov function are considered.

We can notice the effectiveness of the piecewise-polynomial Lyapunov function approach for proving stability even in the presence of sliding modes and parametric uncertainties, finding a more reliable upper bound on the decay rate compared to piecewise-quadratic Lyapunov functions.

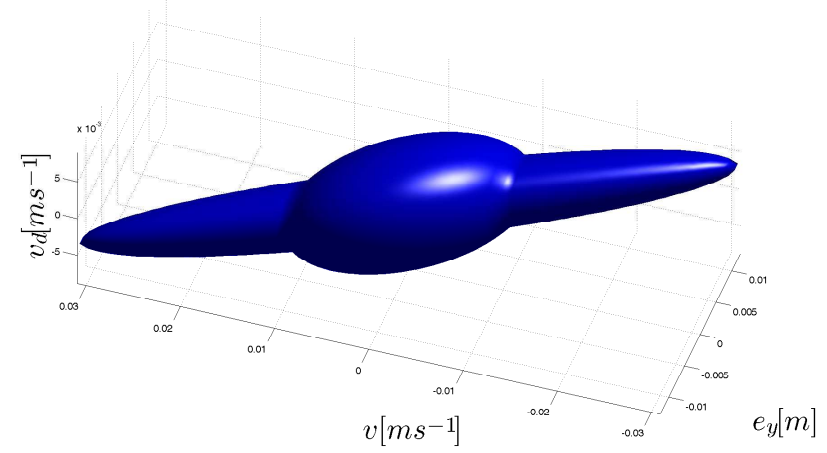

Fig. 17. A Lyapunov function level curve on the $\left(e_{y}, v, v_{d}\right)$ space, friction in relay form, performance study, $m=2$.

\begin{tabular}{|c|c|c|c|}
\hline & $\begin{array}{c}\text { Lyapunov } \\
\text { function degree }\end{array}$ & Stability & Tracking \\
\hline & $m=1$ & $\begin{array}{c}\text { feasible } \\
\text { with } \alpha=0.0897\end{array}$ & feasible \\
\hline & $m=2$ & $\begin{array}{c}\text { feasible } \\
\text { with } \alpha=0.4751 \\
\end{array}$ & feasible \\
\hline$\hat{\imath}$ & $m=1$ & $\begin{array}{c}\text { feasible } \\
\text { with } \alpha=0.0671\end{array}$ & feasible \\
\hline $\begin{array}{l}1 \\
\text { ' }\end{array}$ & $m=2$ & $\begin{array}{c}\text { feasible } \\
\text { with } \alpha=0.4750\end{array}$ & feasible \\
\hline$\uparrow$ & $m=1$ & infeasible & infeasible \\
\hline- & $m=2$ & $\begin{array}{c}\text { feasible } \\
\text { with } \alpha \approx 0\end{array}$ & feasible \\
\hline & $m=1$ & infeasible & infeasible \\
\hline$----\rightarrow$ & $m=2$ & $\begin{array}{c}\text { feasible } \\
\text { with } \alpha \approx 0\end{array}$ & feasible \\
\hline
\end{tabular}

ALL STUDY CASES ON ELECTROPNEUMATIC ACTUATOR

\section{CONClusions}

In this paper, we have investigated the problem of proving the stability of an electropneumatic system in closed-loop with a switching control law modeled as a PWA system with sliding modes due to presence of friction. Some sufficient conditions have been presented for finding piecewise-polynomial Lyapunov functions for PWA systems with sliding modes, uncertainties on parameters and equilibrium sets greater than the mere origin, and potentially on a sliding mode. The new extended conditions successfully found a 4 -th degree piecewise-polynomial Lyapunov function through a convex optimization problem in terms of linear matrix inequalities. We have shown that this method is able to prove stability for a model of a real pneumatic test bench. 


\section{ACKNOWLEDGMENT}

The authors are grateful to Alexandre Trofino Neto (Federal University of Santa Catarina, Brazil) for the quite insightful discussions.

\section{REFERENCES}

[1] O. Ameur, P. Massioni, G. Scorletti, X. Brun, and M. Smaoui. A piecewise-affine approach to the analysis of non-linear control laws for pneumatic systems. In IEEE Conference on Decision and Control, pages 3963-3969, December 2014

[2] O. Ameur, P. Massioni, G. Scorletti, X. Brun, and M. Smaoui. A piecewise-polynomial Lyapunov approach for non-linear switching controllers for pneumatic systems in presence of sliding modes. In Preprint submitted to 54th IEEE Conference on Decision and Control, 2015.

[3] B. Armstrong-Hélouvry, P. Dupont, and C. Canudas De Wit. A survey of analysis tools and compensation methods for the control of machines with friction. In Automatica, volume 30, pages 1083-1138, July 1994.

[4] S. Boyd, L. El Ghaoui, E. Feron, and V. Balakrishnan. Linear Matrix Inequalities in System and Control Theory, volume 15 of SIAM Studies in Applied Mathematics. Society for Industrial and Applied Mathematics, 1994.

[5] M.S. Branicky. Multiple Lyapunov functions and other analysis tools for switched and hybrid systems. In IEEE Transactions on Automatic Control, volume 43, pages 475-482, April 1998.

[6] X. Brun, M. Belgharbi, S. Sesmat, D. Thomasset, and S. Scavarda Control of an electropneumatic actuator, comparison between some linear and non-linear control laws. In Proceedings of the institution of mechanical engineers Part I - Journal of Systems and Control Engineering, volume 213, pages 387-406, 1999.

[7] X. Brun, S. Sesmat, D. Thomasset, and S. Scavarda. Study of "sticking and restarting phenomenon" in electropneumatic positioning systems. In Journal of Dynamic Systems Measurement and Control, volume 127, pages 173-184, May 2005.

[8] X. Brun, D. Thomasset, and E. Bideaux. Influence of the process design on the control strategy: application in electropneumatic field. In Control Engineering Practice, volume 10, pages 727-735, 2002.

[9] C. Canudas De Wit, H. Olsson, K.J. Åström, and P. Lischinsky. A new model for control of systems with friction. In IEEE Transactions on Automatic Control, volume 40, pages 419-425, March 1995.

[10] M.D. Choi, T.Y. Lam, and B. Reznick. Sums of squares of real polynomials. In Proceedings of Symposia in Pure Mathematics, volume 58, pages 103-126, 1995

[11] P. R. Dahl. Measurement of solid friction parameters of ball bearings. In Proceeding of 6th Annual Symposium on Incremental Motion Control System and Devices, pages 49-60, March 1977.

[12] T. Dezuo, L. Rodrigues, and A. Trofino. Stability analysis of piecewise affine systems with sliding modes. In Proc. of the American Control Conference, pages 2005-2010, June 2014.

[13] A.F. Filippov and F.M. Arscott. Differential equations with discontinuous righthand sides: control systems, volume 18. Springer Science \& Business Media, 1988.

[14] L. Fridman, J. Moreno, and R. Iriarte. Sliding Modes After the First Decade of the 21st Century: State of the Art. Lecture Notes in Control and Information Sciences. Springer, 2011.

[15] A. Hassibi and S. Boyd. Quadratic stabilization and control of piecewiselinear systems. In Proceedings of the American Control Conference, volume 6, pages 3659-3664, Juin 1998.

[16] S. Hedlund and M. Johansson. A toolbox for computational analysis of piecewise linear systems. In Proceedings of European Control Conference, 1999.

[17] Z.W. Jarvis-Wloszek. Lyapunov based analysis and controller synthesis for polynomial systems using sum-of-squares optimization. $\mathrm{PhD}$ thesis, University of California, Berkeley, 2003.

[18] M. Johansson. Piecewise linear control systems. Springer, 2003.

[19] M. Johansson and A. Rantzer. Computation of piecewise quadratic Lyapunov functions for hybrid systems. In IEEE Transaction in Automatic Control, volume 43, pages 555-559, April 1998.

[20] D. Karnopp. Computer simulation of stick-slip friction in mechanical dynamic systems. In Journal of Dynamic Systems, Measurement and Control, volume 107, pages 100-103, March 1985.

[21] H.K. Khalil. Non Linear Systems, Third Edition. Prentice Hall, 2002.

[22] S. Krishnamurthy and J. Lee. A computational stability analysis of discrete-time piecewise linear systems. In IEEE Conference on Decision and Control and 28th Chinese Control Conference, pages 1106-1111, December 2009
[23] M. Moarref and L. Rodrigues. Asymptotic stability of piecewise affine systems with sampled-data piecewise linear controllers. In IEEE Conference on Decision and Control and European Control Conference (CDC-ECC), pages 8315-8320, December 2011.

[24] P.A. Parrilo. Semidefinite programming relaxations for semialgebraic problems. In Mathematical programming, volume 96, pages 293-320, May 2003.

[25] N. B. O. L. Pettit and E. P. Wellstead. Analyzing piecewise linear dynamical systems. In IEEE on Control Systems, volume 15, pages 43-50, 1995.

[26] B. Samadi and L. Rodrigues. A unified dissipativity approach for stability analysis of piecewise smooth systems. In Automatica, volume 47, pages 2735-2742, December 2011.

[27] M. Smaoui, X. Brun, and D. Thomasset. High order sliding mode for an electropneumatic system: a differentiator-controllers design. In International Journal of Robust and Nonlinear Control, volume 18 , pages 481-501, 2008.

[28] K. Turki, M. Smaoui, D. Thomasset, and X. Brun. A solution to the "stick-slip" problem for an electropneumatic drive. In International Journal of Fluid Power, volume 12, pages 19-29, January 2011.

[29] A. Tustin. The effect of backlash and speed-dependent friction on the stability of closed-cycle control systems. In Journal of the Institution of Electrical Engineers, volume 94, pages 143-151, January 1947.

[30] J. Xu and L. Xie. Homogeneous polynomial Lyapunov functions for piecewise affine systems. In American Control Conference, volume 1, pages 581-586, June 2005.

[31] T. Yoshizawa. Stability theory by Liapunov's second method, volume 9. Mathematical Society of Japan, 1966.

[32] A.L. Zelentsovsky. Nonquadratic Lyapunov functions for robust stability analysis of linear uncertain systems. In IEEE Transactions on Automatic Control, volume 39, pages 135-138, January 1994. 


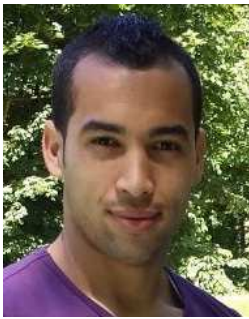

Omar Ameur got his diploma of engineer from Ecole Nationale Polytechnique of Algiers, Algeria, in 2011, and his M.Sc. in Control Engineering from University of Lyon 1, France, in 2012. From October 2012 to November 2015, he has been a $\mathrm{Ph} . \mathrm{D}$. candidate in Laboratoire Ampère at Ecole Centrale de Lyon, in France, and he is currently employed in the industry as a research engineer. Dr. Ameur's main research interests are nonlinear control of pneumatic actuators and the analysis of piecewise affine systems.

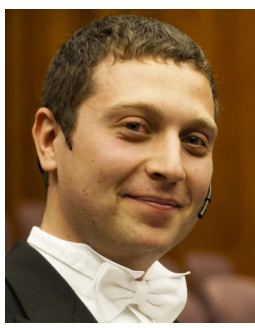

Paolo Massioni got his M.Sc. in Aerospace Engineering from Politecnico di Milano, Italy, in 2005, and his Ph.D. in Control Engineering from Delft University of Technology, the Netherlands, in 2010. From 2012 he is assistant professor at Laboratoire Ampère at the French National Institute for Applied Science (INSA) of Lyon, France. Dr. Massioni has been visiting scientist in the European Space Research and Technology Center (ESTEC), Noordwijk, the Netherlands, and he is a past recipient of a Rubicon grant from the Netherlands Organisation for Scientific Research (NWO) and the Marie Curie COFUND Action. His main interests are distributed control, adaptive optics, and nonlinear systems performance.

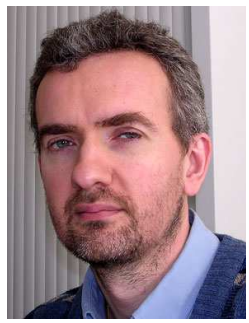

Gérard Scorletti received the engineering degree from the Ecole Nationale Supérieure de Techniques Avancées (ENSTA), Paris, France, and obtained his Ph.D. degree in automatic control from Orsay University, France, in June 1997. He has been a Research Associate with the Centre for Systems Engineering Applied Mechanics, Belgium, from 1997 to 1998 . From 1998 to 2007, he has been an assistant professor of Electrical Engineering at Caen University, France, and he held a permanent joint appointment with the GREYC. Currently, he is a professor of Automatic Control at Ecole Centrale de Lyon, France, with a permanent joint appointment with the Ampère Laboratory. His research interests include robust and LPV control, nonlinear control, identification for control and linear matrix inequality applications with a special emphasis on CAD.

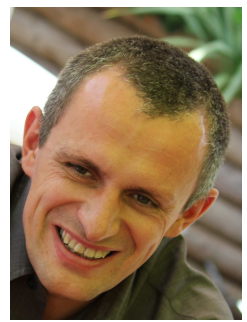

Xavier Brun was born in France in 1973. He received the Ph.D. degree from the French National Institute for Applied Science (INSA) of Lyon, France, in 1999. He became an Associate Professor with the Laboratoire d'Automatique Industrielle, INSA Lyon, in 2001. Since 2011, he has been a Professor at the Laboratoire Ampère, INSA Lyon, France. His current research interests include control for mechatronic and fluid power systems.

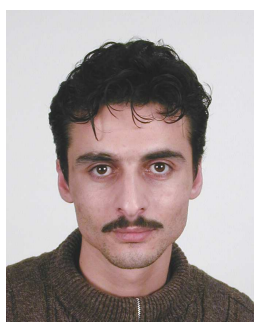

Mohamed Smaoui was born in Sfax, Tunisia, in 1976. He received the Electrical Engineering degree from Ecole Nationale des Ingénieurs de Sfax, Tunisia, in 2000, and the M.Sc. and the Ph.D. degrees in automation from INSA Lyon, France, in 2001 and 2004 respectively. He has been an assistant professor at the department of Mechanical Engineering at INSA Lyon since 2005. His research interests include advanced control of mechanical systems. 